\title{
Development of Meter-Scale U-Shaped and O-Shaped Oscillating Heat Pipes for GAPS
}

\author{
Shun Okazaki*,‡, Hideyuki Fuke*,§, Yoshiro Miyazaki ${ }^{\dagger, \uparrow}$ and Hiroyuki Ogawa*,\| \\ *Japan Aerospace Exploration Agency (JAXA) ISAS \\ Sagamihara-City, Kanagawa 252-5210, Japan \\ ${ }^{\dagger}$ Fukui University of Technology, Fukui-City \\ Fukui 910-8505, Japan \\ 市kazaki.shun@jaxa.jp \\ Sfuke.hideyuki@jaxa.jp \\ Imiyazfam@lapis.plala.or.jp \\ "Iogawa.hiroyuki@jaxa.jp
}

Received 2014 June 30; Revised 2014 August 31; Accepted 2014 September 1; Published 2014 October 27

\begin{abstract}
A meter-scale Oscillating Heat Pipe (OHP) has been developed for the General Anti-Particle Spectrometer (GAPS) project. Two types of OHP routing, U-shaped and O-shaped, have been investigated. For the operation at low temperature, R410A was used as the working fluid. As the result of the investigation, we verified for the first time that both the meter-scale U-shaped and O-shaped OHPs can transfer heat under gravity in a wide temperature range between $20^{\circ} \mathrm{C}$ and $-60^{\circ} \mathrm{C}$. Generally, the Oshaped OHP showed better performance than the U-shaped OHP. Both OHP models showed good thermal conductance and a good amount of heat transport under the particular sets of conditions which meet the design requirements. In order to clarify the drive force to operate OHP to further improve the OHP design, the performance difference between the U-shaped and the O-shaped models has been interpreted in terms of the gravity effect and the pressure loss.
\end{abstract}

Keywords: Oscillating Heat Pipe (OHP), balloon-craft thermal design, cooling system, full-scale model, GAPS.

\section{Introduction}

General Anti-Particle Spectrometer (GAPS) is a US-Japan cooperative balloon-borne space-science project, which aims to contribute to solving the dark matter mystery through highly sensitive antiparticle cosmic-ray observation (Hailey et al., 2013). One of the most important technical components to be developed for GAPS is a system to cool the core detectors efficiently (Fig. 1). In the GAPS full-scale design, 4000 detectors are arranged in 13 layers, which are spread over a wide space of $2 \mathrm{~m} \times 2 \mathrm{~m} \times 2 \mathrm{~m}$, and their total heat dissipation is about $800 \mathrm{~W}$. That is, the heat source has a feature that the total amount of heat is large but the heat flux is low. To ensure good detector performance, it is required to cool the detectors down to lower than $-35^{\circ} \mathrm{C}$, which is nearly the ambient temperature at balloon flight altitudes. The $800 \mathrm{~W}$ heat generated by the detectors must be transported to a radiator attached to the payload sidewall, which is $2 \mathrm{~m}$ from the detectors on average, and must be dissipated to space by heat radiation. The radiator is expected to be cooled down to around $-50^{\circ} \mathrm{C}$. The heat transfer route between the detectors and the radiator must minimize its mass in the field of view of the detectors. Because the balloon flight environment is under gravity and because the heat transfer route must weave through detectors, the heat must be transferred under gravity. Because this is a flight experiment, the power consumption and the weight of the thermal control system must be minimized. 


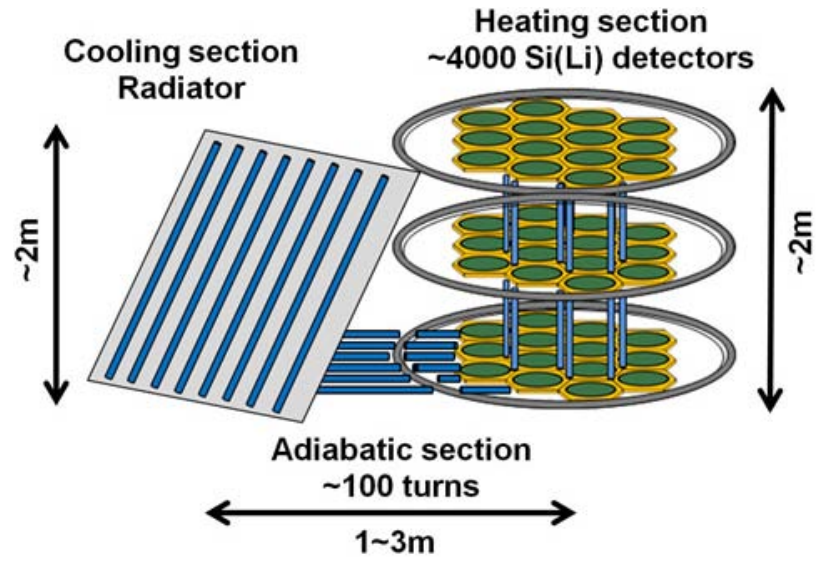

Fig. 1. Conceptual diagram of the GAPS cooling system in the case of the U-shaped heat transfer route.

An option to minimize the mass of transfer tubes within the detector view is to gather the detector heat dissipation once in the vertical direction and then transfer it horizontally to the payload sidewall. Following this direction, the original design of the heat transfer route is " $U$ shaped." The two vertical sides of the U-shape correspond to the stack of detectors (the heat input section, or "the heating section") and the radiator (the heat dissipation section, or "the cooling section"), and the horizontal side corresponds to the horizontal heat transfer part (or "the adiabatic section"). The heat transfer lines in the heating section pass through all the 13 layers, and at each cross-point, every line absorbs the heat dissipation corresponding to three detectors. Therefore, in this design, about 100 vertical heat transfer lines are arranged in the heating section. The heat transport capability per line is required to be larger than $8 \mathrm{~W}$ within the thermal gradient of $15^{\circ} \mathrm{C}$ (between the heating section of $-35^{\circ} \mathrm{C}$ and the cooling section of $-50^{\circ} \mathrm{C}$ ).

\section{Oscillating Heat Pipe (OHP)}

One prospective method to meet the thermal requirements described in the previous section is to use the closed-loop single-phase-fluid pumping method. Technically this method is feasible, but the pump needs non-negligible amount of electric power and the heat transfer tube must be thick (resulting in large mass). To overcome these weaknesses, we have developed an alternative challenging method using the Oscillating Heat Pipe (OHP) technique (Fuke et al., 2014; Mognet et al., 2014).
The Oscillating Heat Pipe (OHP) is a novel technique to realize the heat transfer with very low power consumption through a passive thermo-fluiddynamics process. An OHP consists of a meandering closed (or pressurized) capillary tube going back and forth multiple times between a heating section and a cooling section. Basically, the OHP operation relies on the oscillation and/or the circulation of vapor plugs and liquid slugs in the capillary tube. Vapor bubbles are generated and grow in the heating section, and then collapse in the cooling section. The generation and the collapse of vapor bubbles pump the liquid slugs, which cause the pressure and temperature fluctuations. Compared to the conventional heat pipe, the OHP has many advantages such as simple fabrication capability (because the OHP's capillary tube needs no internal wick), lower sensitivity to its orientation (or gravity), capability for a large amount of heat transfer, and adjustability to low heat flux (Akachi et al., 1996). In addition, by utilizing a liquid reservoir, the OHP can enhance its potential by serving as a temperature controllable device (Iwata et al., 2011). The temperature of the heating section can be controlled by controlling the liquid reservoir temperature. The reservoir is also useful to keep the amount of the effective working fluid by compensating for possible tiny leaks.

Since the OHP has been researched mainly to understand its fundamental phenomena, most of the OHPs studied in the past were desktop-scaled less than $1 \mathrm{~m}$ with a planar routing and were operated at room temperature. Thus, we have researched the suitability of the OHP for GAPS step by step. At first, as a basic study, we developed a scaleddown U-shaped OHP model with a turn length of $1 \mathrm{~m}$. As the result, we verified for the first time both that the OHP can be operated with a threedimensional routing and that the OHP can be operated at low temperatures around $-50^{\circ} \mathrm{C}$ by using R410A (a material alternative for chlorofluorocarbon (Nagashima, 2008)) as the working fluid (Fuke et al., 2014; Mognet et al., 2014; Okazaki et al., 2012a).

As the next step, we developed a U-shaped OHP model whose turn length is of average size $6 \mathrm{~m}$, consisting each of $2 \mathrm{~m}$ long heating section, adiabatic section, and cooling section. With this model, we verified for the first time that a large U-shaped OHP can function at least at room temperature (Okazaki et al., 2012b). However, at low 
temperatures its performance was not as good as it was at room temperature. The temperature dependence of the performance degradation was more obvious than the case of the scaled-down U-shaped OHP. We considered that this performance degradation at low temperatures is mainly caused by gravity. To clarify the gravity effect as well as to seek a way to improve the thermal performance, a modified heat transfer route "O-shaped," which has an additional adiabatic section bridging the top of two vertical sides, has been investigated. In this paper, thermal performances of a 6-m U-shaped OHP model and an 8-m O-shaped OHP model have been investigated, followed by an interpretation of the gravity effect.

\section{Experimental Setup}

Figure 2 illustrates setups of the tested U-shaped and O-shaped OHPs with thermocouples. The test specimen consisted of a closed-loop capillary tube with a liquid reservoir. The design parameters, such as the reservoir connection point, the working fluid material, the tube diameter, and the check valve locations, were applied as they were optimized through the study of the scaled-down 1-m U-shaped OHP model described in the previous section. The reservoir was connected to the OHP adiabatic section near the heating section inlet. As the working fluid, R410A was used. The capillary tube is made with copper, and its inner and outer diameters are 1.0 and $1.6 \mathrm{~mm}$, respectively. The lengths of the heating, cooling, and adiabatic sections are $2 \mathrm{~m}$ each. The amount of R410A enclosed in the OHP was about $350 \mathrm{~g}$, which corresponds to a filling ratio at $25^{\circ} \mathrm{C}$ of $67 \%$ for the U-shaped model and $60 \%$ for the O-shaped model.

The number of heat transfer lines between the heating section and the cooling section was scaled down to 16 from the full-scale number of 100. As for the U-shaped OHP, one tube turn was assigned to each line. That is, the U-shaped model has 16 turns (in other words, 32 parallel tubes were aligned in each section). The O-shaped OHP was modified from the U-shaped model by bridging the two vertical sides. Thus, the number of "loops" between the heating section and cooling section is 32 . The length of a turn of the U-shaped OHP is $12 \mathrm{~m}$ and the length of a loop of the O-shaped OHP is $8 \mathrm{~m}$. The total lengths of the tube of the U-shaped and the O-shaped OHPs are $192 \mathrm{~m}$ and $256 \mathrm{~m}$, respectively.

Sixteen check valves were installed alternately in the adiabatic section tubes to constrain the working-fluid flow to one direction. It is known that the one-direction circulation flow provides higher heat transfer capability to OHPs than the oscillation flow. In the heating section of the U-shaped model, the working fluid flows up to the top of the heating section and then flows down towards the adiabatic section. In the case of the O-shaped heating section, the working fluid always flows upward from bottom to top. The capillary tubes in the heating and the adiabatic sections were

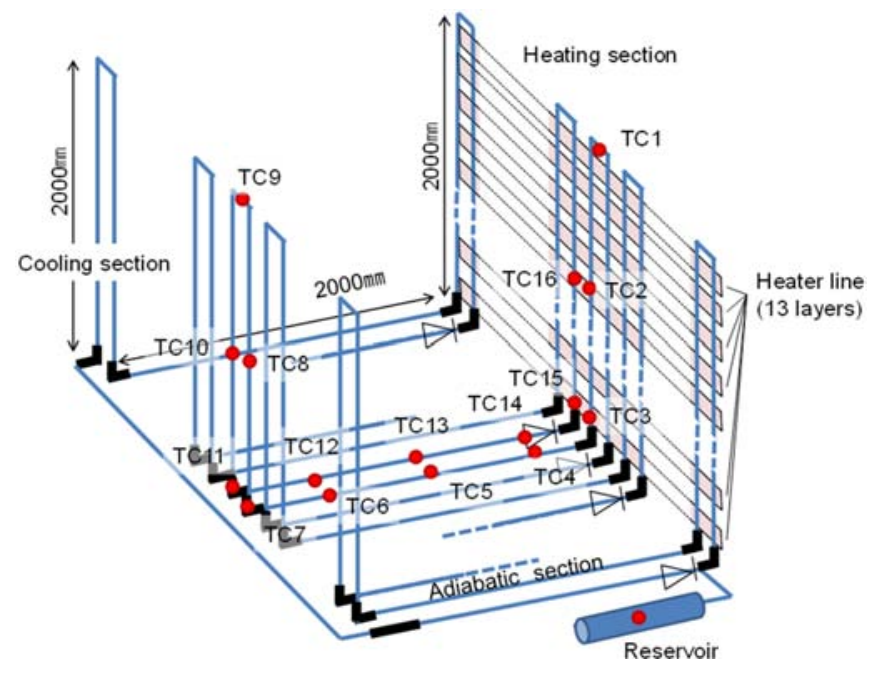

U-shaped OHP

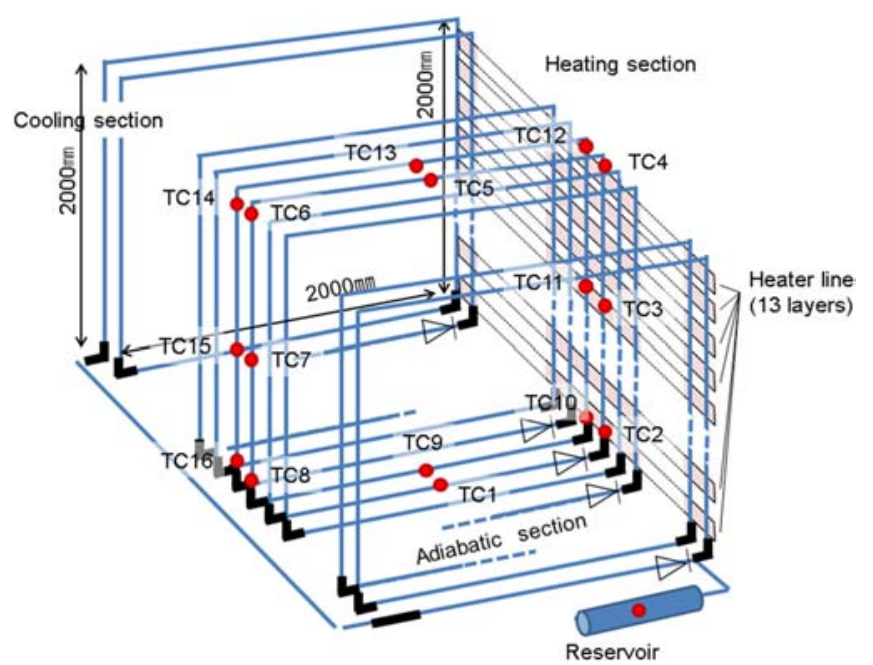

O-shaped OHP

Fig. 2. (Color online) Schematic illustration of the U-shaped (left) and the O-shaped (right) OHP models. Thermocouple locations are shown by red circles. 
thermally insulated from other components by a Styrofoam insulator. Additionally, the tubes in the U-shaped cooling section were alternately insulated so that the upward flows are insulated (not cooled) and the downward flows are cooled (not insulated). This alternate insulation is expected to make the flow more efficient, because the upward flow in the anti-gravity direction is desired to be vapor whose density is lower than the liquid density.

Thirteen heater sheets were attached to the heating section to simulate the heat source distribution of the GAPS detectors. The liquid reservoir was a stainless steel tank with a volume capacity of $3.15 \times 10^{-4} \mathrm{~m}^{3}$. A Kapton heater was attached to the reservoir and was controlled by a feedback controller, which controls the heat input to the heater to keep the reservoir temperature typically $10^{\circ} \mathrm{C}$ higher than the cooling section temperature. Throughout all tests, the power consumption of the reservoir heater was always small (less than $10 \mathrm{~W}$ ). The temperatures at various points of the OHP models were measured by T-type thermocouples and were monitored and recorded every second by a data logger. In this paper, the temperature data of the central turns (or the central loop) is used as the representative OHP temperatures. All tests were conducted in a thermostatic chamber at atmospheric pressure. The radiator cooling was simulated by convection instead of the radiative cooling used in actual flight.

\section{Thermal Performance of the U-Shaped OHP}

Figures 3 and 5 show the time variation of the U-shaped OHP temperature data, when the cooling section temperature was kept at $20^{\circ} \mathrm{C}$ and $-50^{\circ} \mathrm{C}$, respectively. Figures 3(a) and 5(a) correspond to temperature profiles of the central turn tube, which has a check valve, and Figs. 3(b) and 5(b) correspond to those of the neighboring tube without check valve. Figures 4 and 6 show the temperature distributions along the central turn when the OHP operation was at steady state.

The total heat input to the heating section was first set at $50 \mathrm{~W}$. However, different from the O-shaped OHP described in the following section, the U-shaped OHP model did not start operation at $50 \mathrm{~W}$. Therefore, in Figs. 3 and 5, the heat input was first set at $100 \mathrm{~W}$ for the start-up, and then increased up to $200 \mathrm{~W}$ in $50 \mathrm{~W}$ increments.

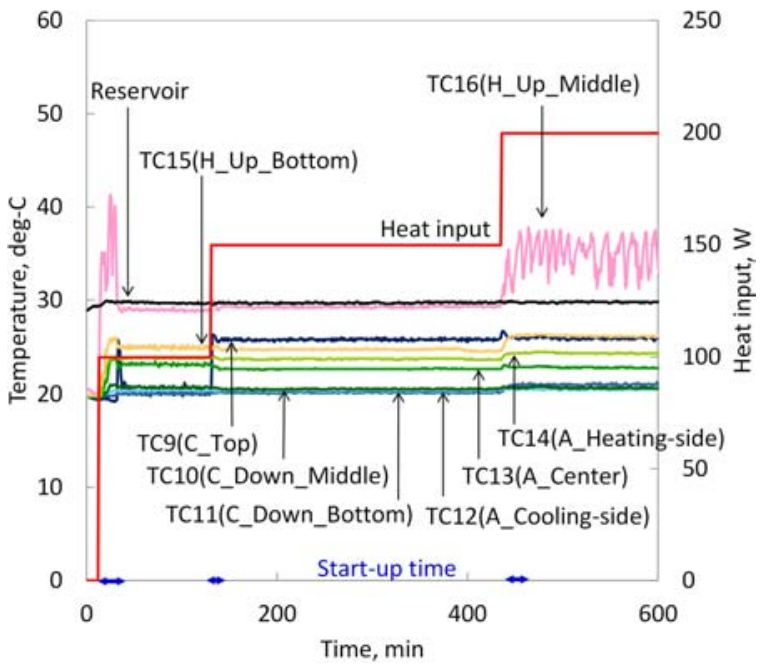

(a) Tube with a check valve

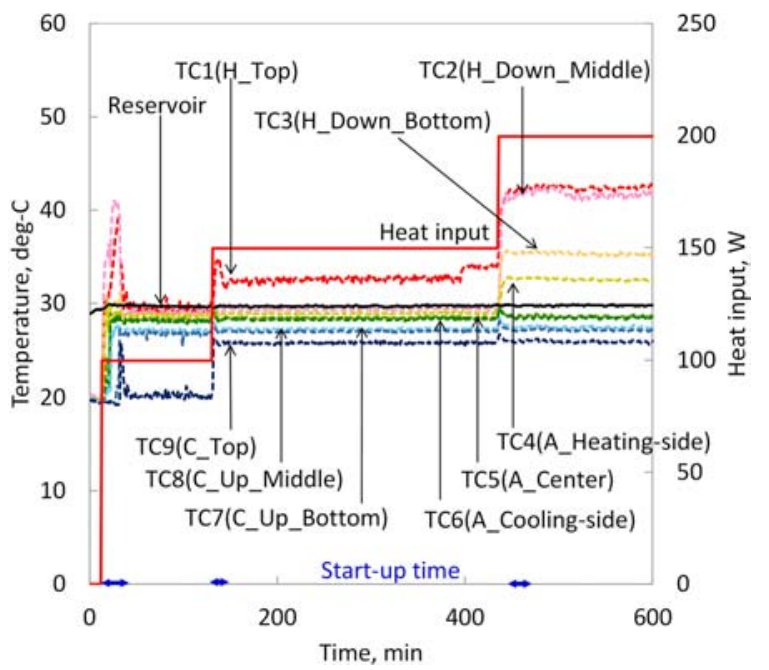

(b) Tube without check valve

Fig. 3. Time variation of temperatures of the U-shaped OHP at central turn tubes (a) with a check valve and (b) without check valve at $20^{\circ} \mathrm{C}$.

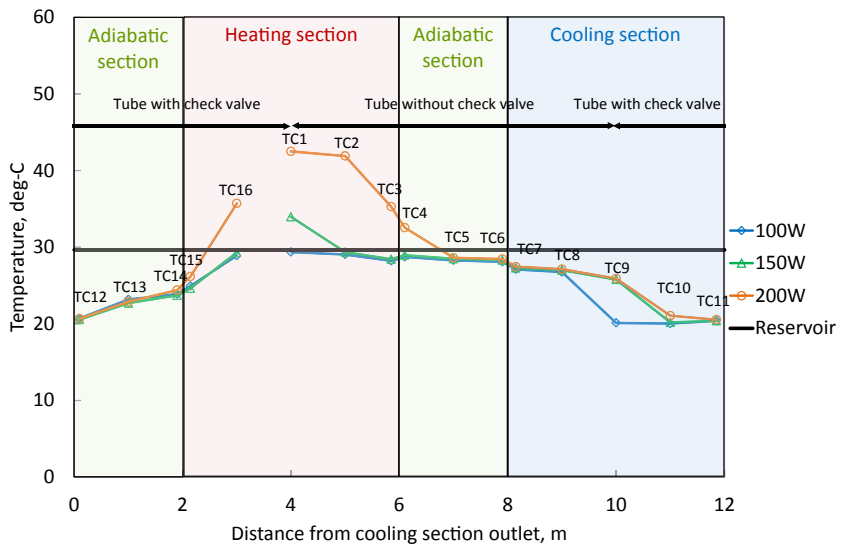

Fig. 4. Steady state temperature distribution along the central turn of the U-shaped OHP model at $20^{\circ} \mathrm{C}$. 


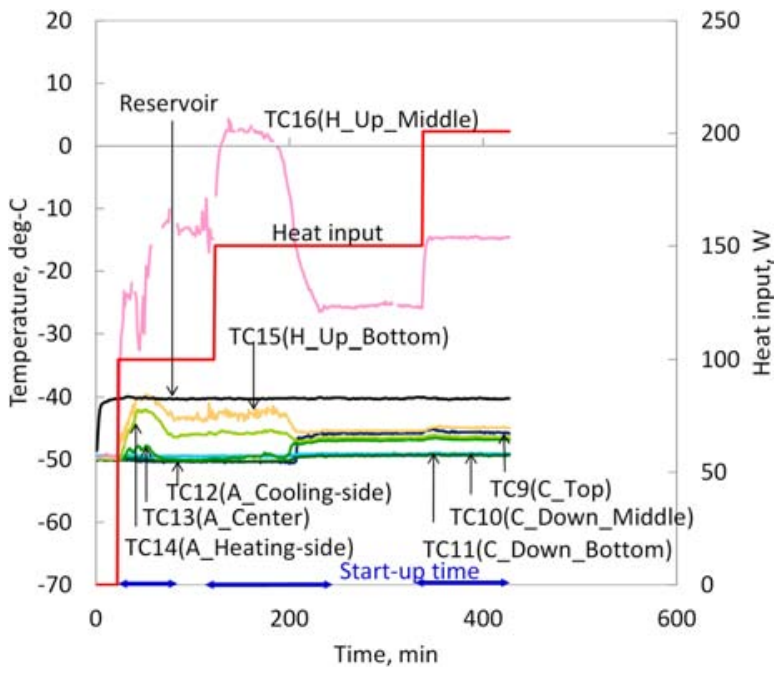

(a) Tube with a check valve

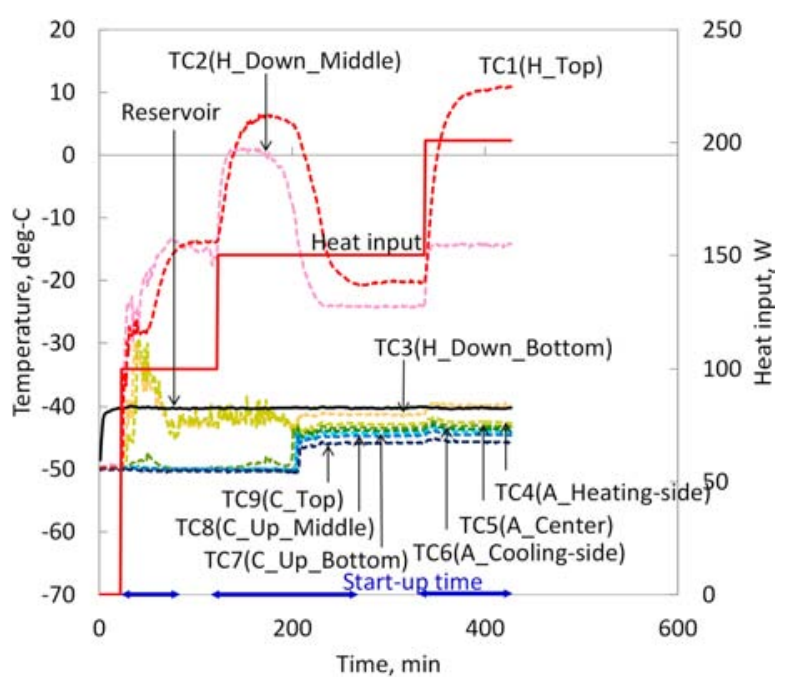

(b) Tube without check valve

Fig. 5. Time variation of temperatures of the U-shaped OHP at central turn tubes (a) with a check valve and (b) without check valve at $-50^{\circ} \mathrm{C}$.

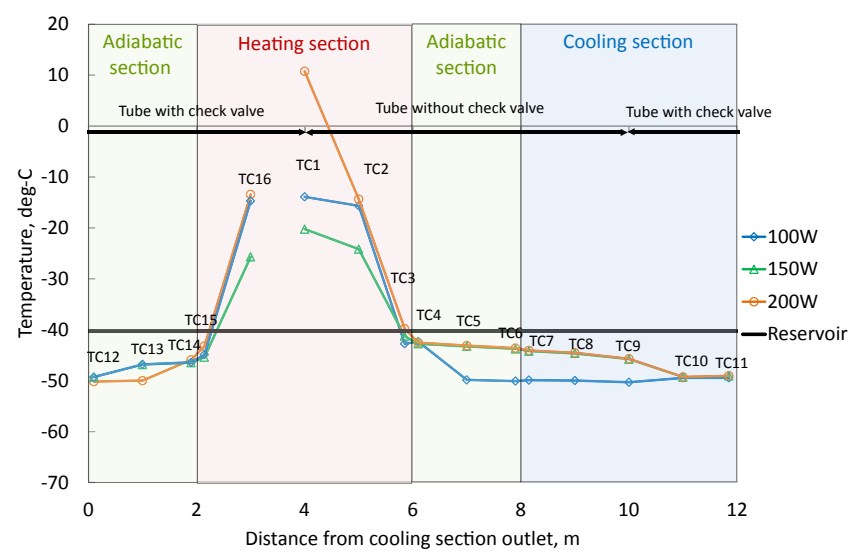

Fig. 6. Steady state temperature distribution along the central turn of the U-shaped OHP model at $-50^{\circ} \mathrm{C}$.

\subsection{U-shaped $\mathrm{OHP}$ at $20^{\circ} \mathrm{C}$}

In the case of $20^{\circ} \mathrm{C}$, as shown in Fig. 3, a steep increase of the heating section temperature (TC1, TC2 and TC16) was followed by a sharp decrease to around $30^{\circ} \mathrm{C}$, and then within about $20 \mathrm{~min}$ the U-shaped OHP got into a state of steady operation. For the start-up of the OHP, the vapor pressure plays an important role to push the liquid up through the cooling section against gravity. Because the higher the temperature is, the higher the vapor pressure becomes, the increase of the heating section temperature drove the startup breakthrough on the working-fluid flow. At steady state, the heating section temperature was almost the same as the reservoir temperature as expected, because the heating section temperature was induced to be close to the reservoir temperature, which was controlled to be constant.

When the heat load was increased to $150 \mathrm{~W}$, the temperature at the top of the heating section (TC1 in Fig. 3) rose. This temperature rise indicates that a local drying-out occurred in the capillary tube. It seems this local dried-out area expanded to the middle of the heating section (TC2, TC16) when the heat input was increased to $200 \mathrm{~W}$. As shown by Fig. 4, the temperatures in the insulated cooling section tube where the inside vapor rises up (TC7, TC8) were almost same as the temperatures in the adiabatic section (TC5, TC6). It means the working fluid in this insulated tube was not condensed. On the other hand, the temperatures in the uninsulated cooling section tube (TC9, TC10 and TC11 in Fig. 4) show a downward trend toward the downstream direction, which indicates that the working fluid was condensed in this tube during falling down. The common temperature distribution of the cooling section tube (TC7, TC8, TC9, TC10 and TC11) between heat loads of $150 \mathrm{~W}$ and $200 \mathrm{~W}$ suggests that this OHP model reached a limit of heat transportation from the heating section to the cooling section. In other words, the maximum amount of heat transport of this OHP model is around $150 \mathrm{~W}$ at $20^{\circ} \mathrm{C}$. Actually, the increase of the heating section temperature from $150 \mathrm{~W}$ to $200 \mathrm{~W}$ corroborates this.

\subsection{U-shaped $\mathrm{OHP}$ at $-50^{\circ} \mathrm{C}$}

In the case of $-50^{\circ} \mathrm{C}$, as shown in Fig. 5, a steep increase of the heating section temperature (TC1, TC2 and TC16) was observed at the start-up with $100 \mathrm{~W}$ heat load. As shown in Fig. 6, with $100 \mathrm{~W}$ 
heat load, the temperatures in the cooling section (TC10, TC11) were almost the same as the adiabatic section temperature (TC5). We presume that the OHP operated locally. That is, the working fluid oscillated only between the adiabatic section and the heating section. Little heat transport can be expected from this local oscillation.

Back to Fig. 5, when the heat input was increased to $150 \mathrm{~W}$, the temperature at the top and middle of the heating section (TC1, TC2 and TC16) first rose and then descended to a steady state 140 min later simultaneously with the increase of the cooling section temperature (TC9). This means the OHP took $140 \mathrm{~min}$ for the start-up. However, these heating section temperatures were still higher than the reservoir temperature at the steady state. We consider that $150 \mathrm{~W}$ somewhat exceeds the maximum amount of heat transport at $-50^{\circ} \mathrm{C}$ and caused local drying-out around the top and the middle of the heating section. The common temperature distribution of the cooling section (TC7, TC8, TC9, TC10 and TC11) in Fig. 6 between $150 \mathrm{~W}$ and $200 \mathrm{~W}$ also indicates that the OHP reached the transport limit at around $150 \mathrm{~W}$. Actually, the heating section temperatures increased with the heat load increase.

\section{Thermal Performance of the O-Shaped OHP}

Figures 7 and 9 show the time variation of the O-shaped OHP temperature data, when the cooling section temperature was $20^{\circ} \mathrm{C}$ and $-50^{\circ} \mathrm{C}$, respectively. Figures 7(a) and 9(a) show the temperature of the central loop, which has a check valve, and Figs. 7(b) and 9(b) show the temperature of the neighboring loop without check valve. Figures 8 and 10 show the temperature distributions along the central two loops when the OHP operation was at steady state. The heat input to the heating section was first set at $50 \mathrm{~W}$ for the start-up, and then was increased up to $200 \mathrm{~W}$ or $250 \mathrm{~W}$ in $50 \mathrm{~W}$ increments.

\subsection{O-shaped $\mathrm{OHP}$ at $20^{\circ} \mathrm{C}$}

In the case of $20^{\circ} \mathrm{C}$, as shown in Fig. 7, a steep increase of the heating section temperatures (TC3, TC4, TC11 and TC12) was followed by a sharp decrease to around $30^{\circ} \mathrm{C}$, and then the $\mathrm{OHP}$ operated stably. As shown in Fig. 8, these temperatures in the heating section (TC3, TC4, TC11 and TC12) were almost the same as the reservoir temperature. These data show the O-shaped OHP model started up with $50 \mathrm{~W}$ heat load. The uniformity of the heating section temperature suggests that the latent heat of the working fluid was used to transfer the heat, because the fluid must have a constant temperature as long as the fluid is saturated regardless of the heat exchange.

When the heat input was increased to $100 \mathrm{~W}$, the temperatures in the middle of the heating section (TC3 and TC11 in Fig. 7) rose. We consider that a single-phase heat transfer (or a heat transfer by sensible heat) by dried-out vapor or

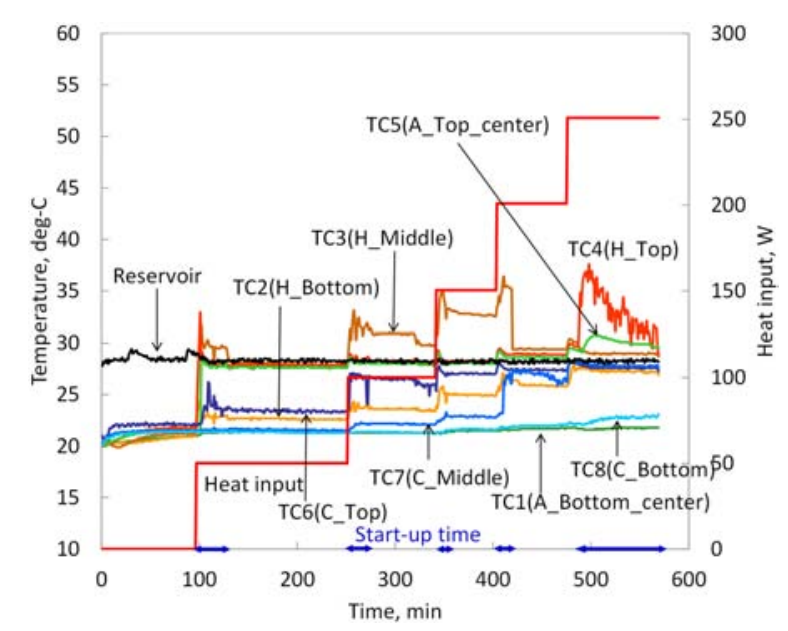

(a) Loop with a check valve

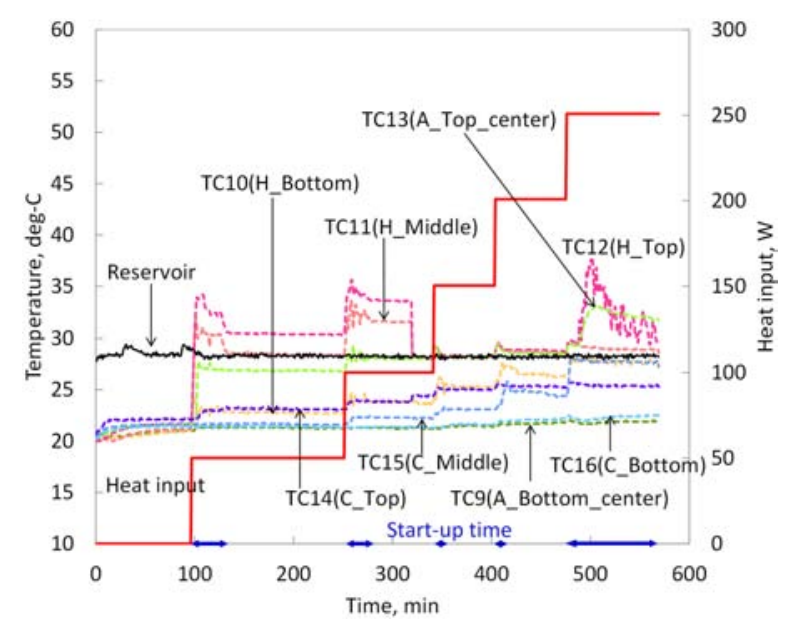

(b) Loop without check valve

Fig. 7. Time variation of temperatures of the O-shaped OHP at a loop (a) with a check valve and (b) without check valve at $20^{\circ} \mathrm{C}$. 


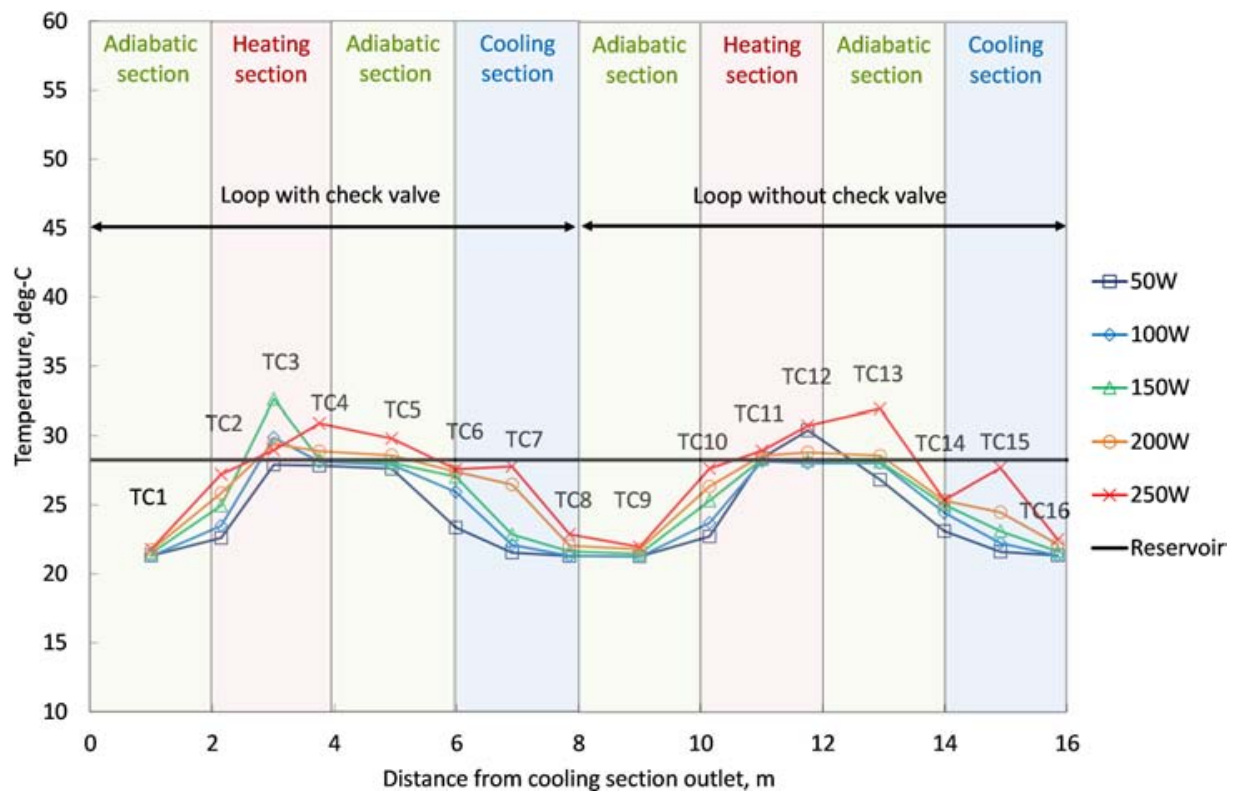

Fig. 8. Steady state temperature distribution along two central loops of the O-shaped $\mathrm{OHP}$ at $20^{\circ} \mathrm{C}$.

pressurized liquid occurred and caused this temperature increase in the middle of the heating section.

When the heat input was increased to $200 \mathrm{~W}$, the heat transfer mode seems to change in the middle of the heating section from the sensible-heat mode to a latent-heat mode. By this mode change, the temperature in the middle of the heating section (TC3) decreased to around the reservoir temperature.

With the $250 \mathrm{~W}$ heat load, the temperatures at the top of the heating section (TC4 and TC12 in Fig. 7) rose above the reservoir temperature. This

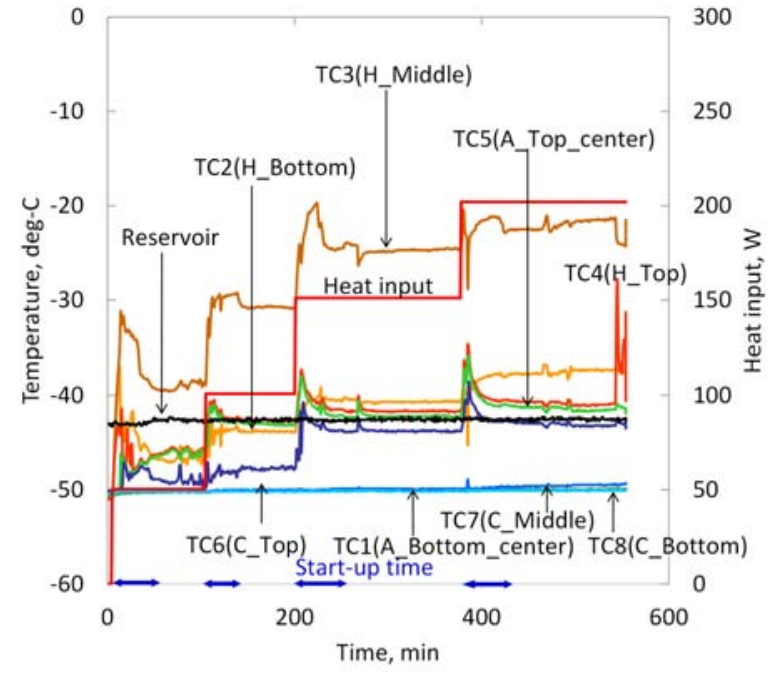

(a) Check valve loop indicates that a local drying-out occurred in the capillary tube at around the top of the heating section.

\subsection{O-shaped $\mathrm{OHP}$ at $-50^{\circ} \mathrm{C}$}

As shown in Fig. 9, in the case of $-50^{\circ} \mathrm{C}$, with $50 \mathrm{~W}, 100 \mathrm{~W}$, and $150 \mathrm{~W}$ heat loads, after a steep increase the heating section temperature (TC2, TC3, TC4, TC10, TC11 and TC12) showed a startup of oscillation followed by a stable OHP operation. During this steady state, as shown by Fig. 10,

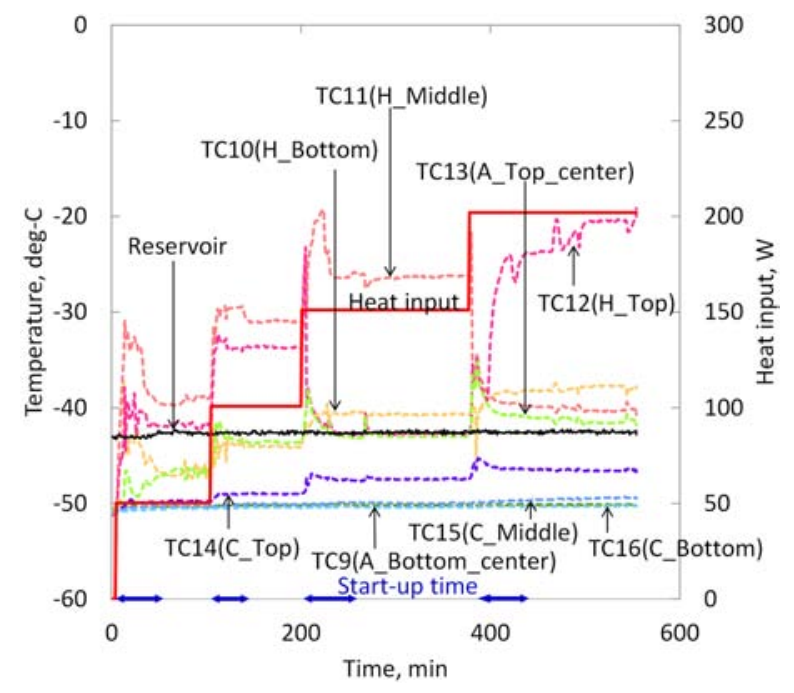

(b) Without check valve loop

Fig. 9. Variation of temperatures of the O-shaped OHP at a loop (a) with a check valve and (b) without check valve at $-50^{\circ} \mathrm{C}$. 


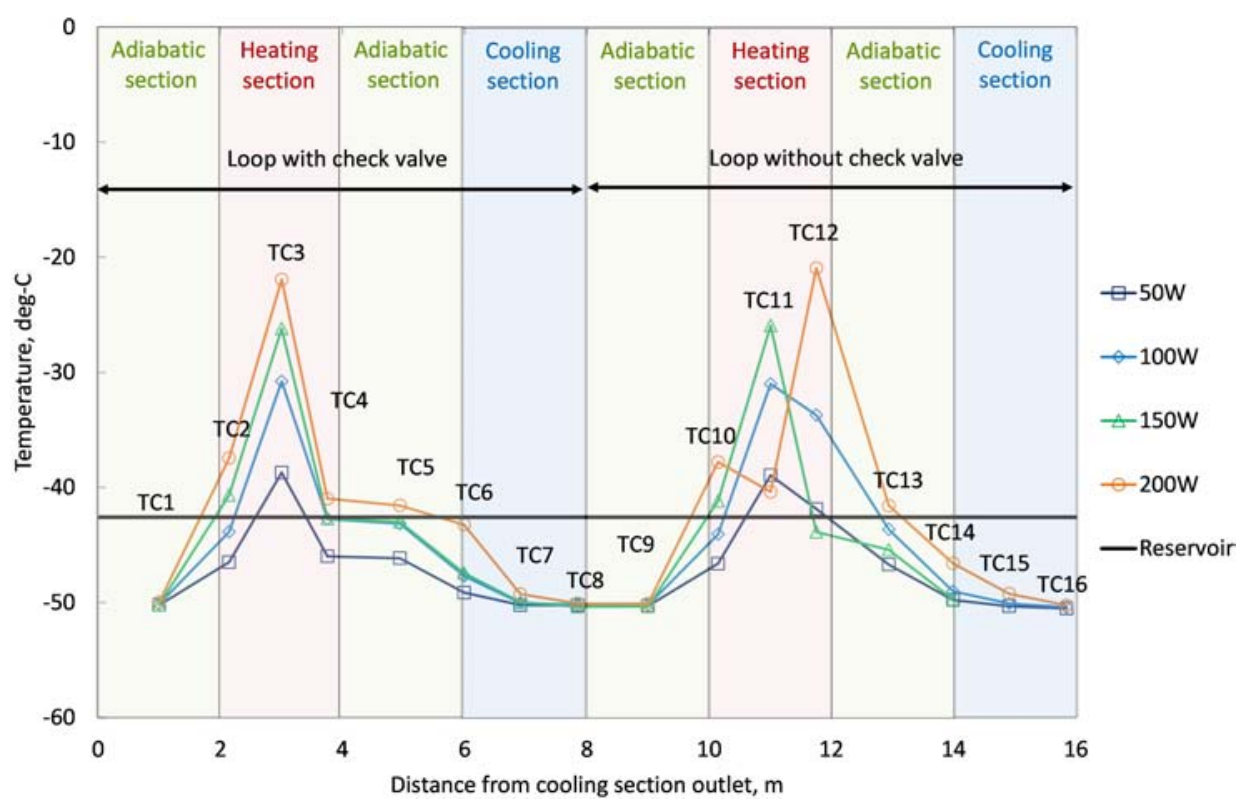

Fig. 10. Steady state temperature distribution along two central loops of the O-shaped $\mathrm{OHP}$ at $-50^{\circ} \mathrm{C}$.

the temperature in the middle of the heating section (TC3, TC11) was higher than the reservoir temperature. This must be caused by a single-phase heat transfer mode.

When the heat input was increased to $200 \mathrm{~W}$, the temperature at the top of the heating section on a loop without check valve (TC12 in Fig. 10) rose. In Fig. 10, the temperature distribution in the cooling section (TC6, TC7, TC8, TC14, TC15 and TC16) were common between $100 \mathrm{~W}$ and $150 \mathrm{~W}$ heat loads. It indicates that the maximum amount of the transportable heat for this O-shaped OHP model at $-50^{\circ} \mathrm{C}$ is around $150 \mathrm{~W}$. Actually, the temperature of the heating section (TC2, TC3, TC4, TC10, TC11 and TC12) increased with the increase of the heat input.

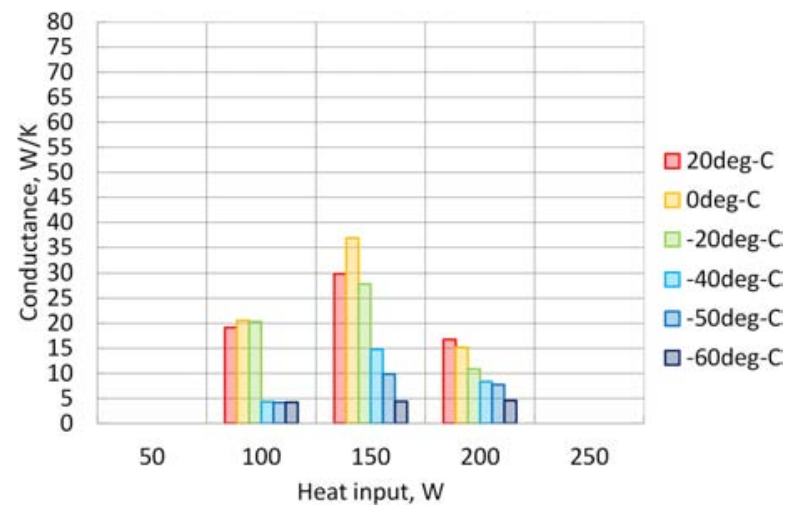

(a) U-shaped OHP

\section{Thermal Conductance}

Figure 11 shows the thermal conductance of the U-shaped and O-shaped OHPs obtained under each cooling section temperature conditions. The thermal conductance was calculated as "the amount of heat input to the heating section divided by the temperature difference between the heating and cooling sections." Generally, the O-shaped OHP shows larger (better) conductance than the U-shaped model. Generally, the thermal conductance increases with increasing temperature, because the higher the temperature is, the steeper the increasing rate of the vapor pressure becomes, which is a drive force to flow the working fluid. Generally, the thermal conductance of the OHP

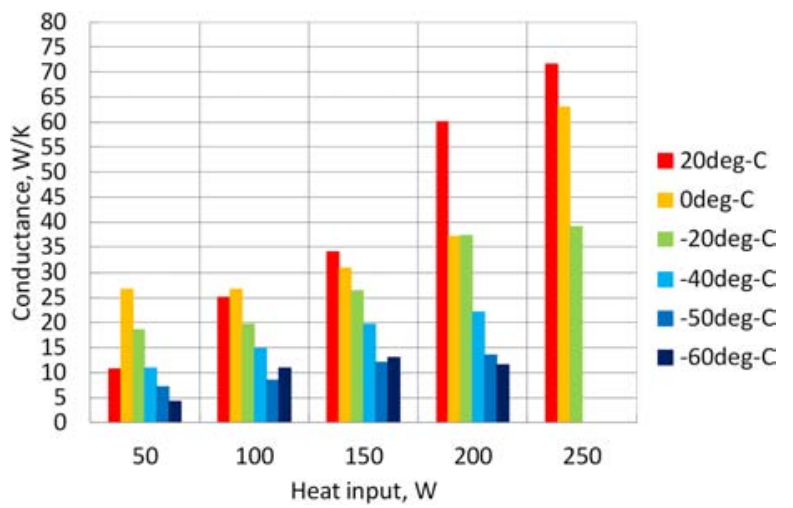

(b) O-shaped OHP

Fig. 11. Thermal conductance of (a) U-shaped and (b) O-shaped OHPs at various temperatures with various heat inputs. 
increases with increasing heat input, because the heating section temperature was induced to be similar to the constant reservoir temperature when the OHP has enough capability to transport the heat load.

However, in the case of the U-shaped OHP at $-60^{\circ} \mathrm{C}$, for example, the thermal conductance was almost constant at only $4 \mathrm{~W} / \mathrm{K}$, which is almost the same as the conductance of the blank test (or the conductance of the bare tube without working fluid). This result shows that the U-shaped OHP did not work as a "heat transfer device" at $-60^{\circ} \mathrm{C}$. Based on the temperature time-variation data and the calculated thermal conductance, we consider that the minimum heat input to start up this $\mathrm{U}$-shaped $\mathrm{OHP}$ was $200 \mathrm{~W}$ at -40 and $-50^{\circ} \mathrm{C}$ and was $100 \mathrm{~W}$ at 20,0 and $-20^{\circ} \mathrm{C}$.

As described in Sec. 1, from the design requirement, the heat transport capability per line should be larger than $8 \mathrm{~W}$ (which corresponds to $128 \mathrm{~W}$ for our scaled-down U-shaped and O-shaped models) at $-50^{\circ} \mathrm{C}$ with a temperature difference between the heating and the cooling sections smaller than $15^{\circ} \mathrm{C}$ (which corresponds to $8.5 \mathrm{~W} / \mathrm{K}$ ). The thermal conductance of the U-shaped and the O-shaped OHP models was about $10 \mathrm{~W} / \mathrm{K}$ and $12 \mathrm{~W} / \mathrm{K}$, respectively, with $150 \mathrm{~W}$ heat input at $-50^{\circ} \mathrm{C}$. Therefore, in terms of the thermal conductance, these OHP models meet the GAPS requirement.

\section{Start-Up Time}

The measured start-up time of the U-shaped and O-shaped OHPs required for reaching a steady state after the heat load increase is shown in Fig. 12. For actual use in GAPS, the shorter the start-up time, the better.
At room temperature, both OHP models show adequate short start-up time. Generally, the startup time increases with an increase of the heat load as well as with a decrease of the temperature. Especially at low temperatures below $-40^{\circ} \mathrm{C}$, the U-shaped model took a longer time to reach a steady state than the O-shaped model.

For the start-up, the vapor generated in the heating section must grow into the cooling section. Because the increasing rate of the saturation vapor pressure is moderate at low temperature, it is reasonable that it takes a longer time for the vapor to grow. In the case of the U-shaped model, the growing vapor must push the liquid up into the cooling section against gravity. Therefore, the U-shaped model is considered to need longer time for the start-up to wait for the heating up of the working fluid that brings a larger temperature gradient or a larger force.

\section{Uniformity of the Heating Section Temperature}

For the actual use in GAPS, the more uniform the heating section temperature, the better. Figure 13 shows the measured average, maximum and minimum temperatures in the heating section at each steady state. In general, the heating section temperature of an OHP with a liquid reservoir is expected to have a uniform heating section temperature at around the reservoir temperature. Actually, the O-shaped OHP showed fairly uniform temperature distributions. On the other hand, the U-shaped OHP showed worse uniformity especially with a heat load of 200 W. As discussed in Sec. 5, $200 \mathrm{~W}$ seems to be above the maximum amount of heat transport of the U-shaped model and thus a

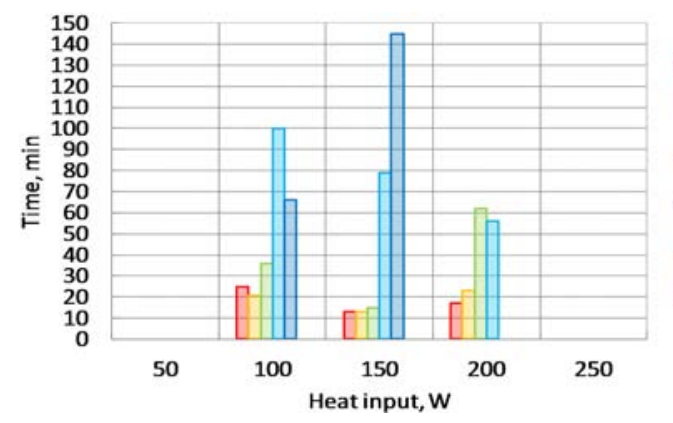

(a) U-shaped OHP

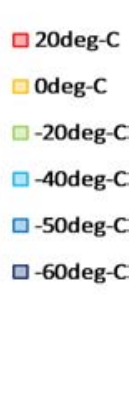

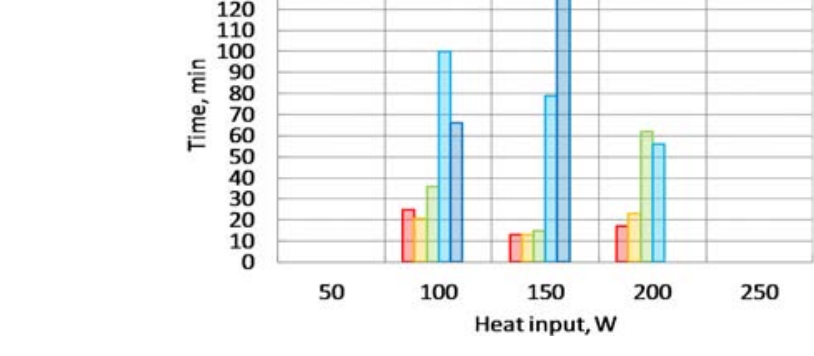

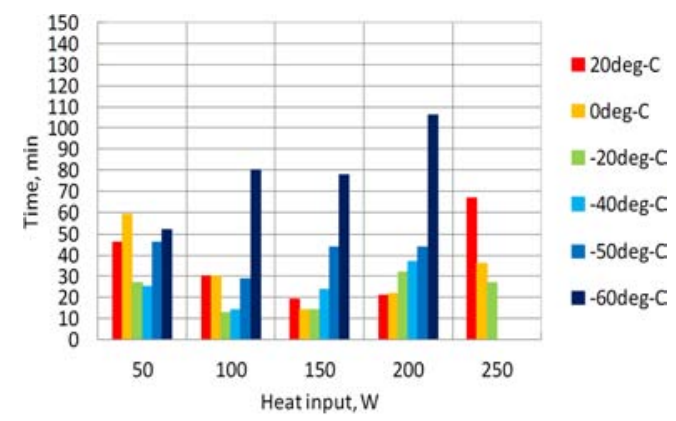

(b) O-shaped OHP

Fig. 12. The start-up time of (a) U-shaped and (b) O-shaped OHPs at various temperatures with various heat inputs. 


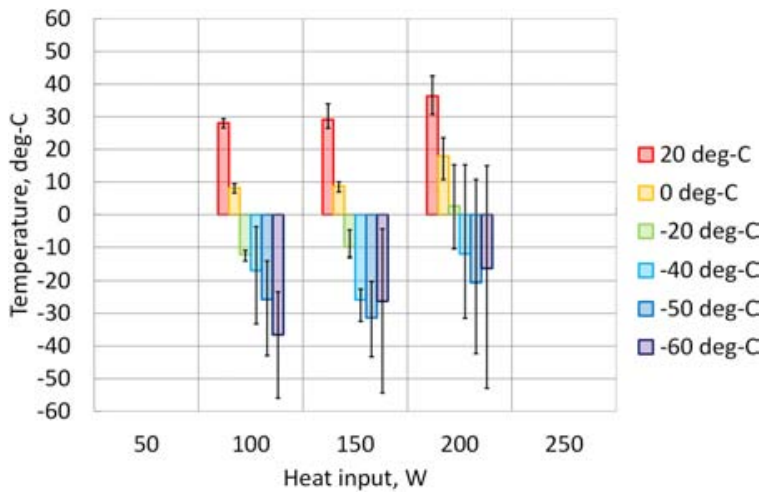

(a) U-shaped OHP

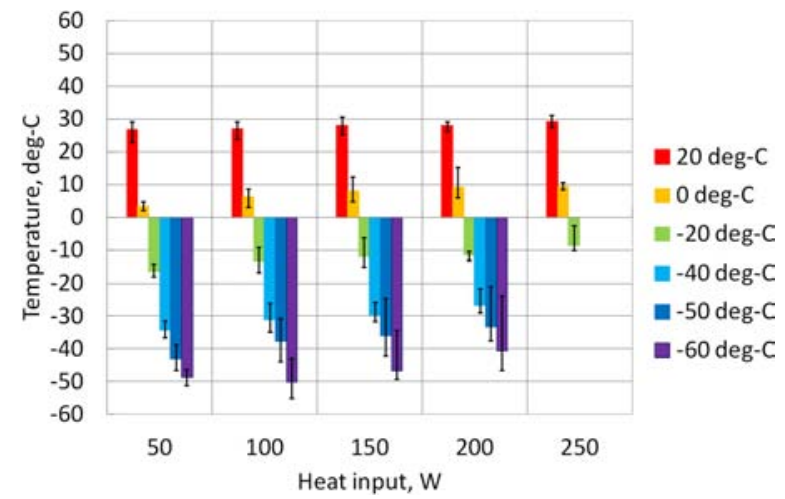

(b) O-shaped OHP

Fig. 13. The average, maximum and minimum temperatures of the heating section at various temperatures and with various heat inputs.

local drying-out at around the top and the middle of the heating section seems to happen. Overload of the heat input breaks the temperature uniformity in the heating section as well as the temperature control of the reservoir.

Although the O-shaped OHP showed better temperature distribution than the U-shaped model, with $150 \mathrm{~W}$ heat load and at $-50^{\circ} \mathrm{C}$, some of the heating section temperatures were above $-35^{\circ} \mathrm{C}$, which is the upper limit of the design requirement. Therefore, in terms of the temperature uniformity, even the O-shaped OHP should be improved.

\section{Interpretation of the Performance Difference Between the U-Shaped and the O-Shaped OHPs}

As discussed in the previous sections, the O-shaped OHP showed generally better thermal performance than the U-shaped model. In this section, to interpret the performance difference between the two models, we estimate the degree of the pressure loss in the capillary tube as well as the degree of the gravity effect on the flow under simple assumptions. Note that the drive force mechanism to operate the OHP, especially for these kinds of meter-scale OHPs, is not theoretically clarified in detail yet. The thermo physical property data of the working fluid R410A was cited from Nagashima (2008).

Under an assumption that all the heat input was used for the evaporation in the heating section and the working fluid flow was circulative in one direction, the working fluid velocity, $v$, needed to absorb all the heat input can be expressed by

$$
v=\frac{Q}{L \rho_{v}} \frac{1}{\pi r^{2}}
$$

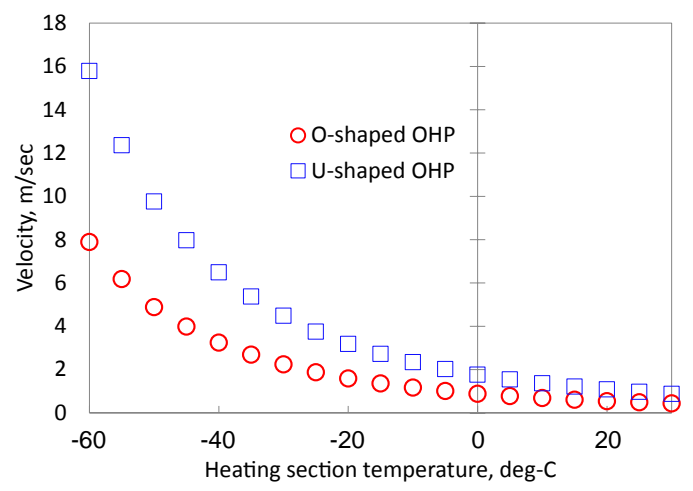

Fig. 14. Assumed velocity of the working fluid needed at the outlet of the heating section to absorb $150 \mathrm{~W}$ heat load.

where $Q, L, \rho_{v}$, and $r$ are the rate of heat input, the amount of latent heat, the liquid density, and the inner radius of the capillary tube, respectively. The heat input $Q$ increases in a staircase pattern when the flow passes each heater line. Figure 14 shows the calculated $v$ at the outlet of the heating section at each temperatures under a condition of $150 \mathrm{~W}$ heat load. At low temperatures $v$ is getting faster, due to the temperature dependence of the R410A physical property. The U-shaped OHP needs faster $v$ than the O-shaped, because the working fluid in the U-shaped model needs to absorb a larger amount of heat per turn for passing heater lines 26 times, while the working fluid in the O-shaped model passes heater lines 13 times per loop. Because the faster the fluid velocity, the higher the pressure loss (or the friction loss) in the capillary tube, the U-shaped section needs a larger drive force than the O-shaped section to circulate the working fluid under the same thermal condition.

From the start-up data, the O-shaped OHP could start heat transfer with lower heat load 
than the U-shaped OHP. The start-up time of the O-shaped OHP was shorter than that of the U-shaped OHP. We consider that the O-shaped OHP utilizes the assist from gravity more effectively than the U-shaped OHP.

It is known that under the slug flow condition, the vapor plug and liquid slug in the OHP capillary tube are separated due to the capillary force, and the plug and slug move together. In a vertical capillary tube, buoyancy is given to a vapor plug that sweeps the liquid in the tube, and the body force is given to a liquid slug due to the gravity. The force direction of the buoyancy and the body force are in opposite direction in a vertical tube under gravity. The buoyancy pushes the working fluid up against gravity and the body force pushes it down in the gravity direction. Thus, the total amount and direction of the force on the slug flow in a vertical capillary tube depend on the volume rate (or the void ratio) of the working fluid. In a vertical tube in the heating section, the void ratio of the working fluid increases in a staircase pattern when the flow passes each heater line and absorbs heat. On the other hand, in a vertical tube in the cooling section, the void ratio decreases continuously when the heat is exhausted through the cooling section. We consider that the unevenness of these forces per unit volume along the turn (or the loop) assists the circulation flow of the working fluid.

When a cooled liquid comes from the cooling section into the heating section, the liquid is heated up to the saturation temperature (which must be around the reservoir temperature) and then vapor plugs must be generated. The mass flow rate of vapor, $\dot{m}_{V}$, in the heating section can be calculated by

$$
\dot{m}_{V}=\frac{Q_{\text {heat }} \alpha-\dot{M} C_{p L}}{L}
$$

where $\dot{m}_{V}, Q_{\text {heat }}, \dot{M}, C_{p L}$, and $\alpha$ are the mass flow rate of vapor, the amount of heat given to a capillary tube by a heater line, the total mass flow rate, the liquid thermal capacity, and the number of heater lines that the working fluid passes, respectively.

When a warm saturated vapor plug comes from the heating section into the cooling section, the vapor plug must be collapsed, condensed, and reduced by the heat dissipation. The mass flow rate of vapor, $\dot{m}_{V}$, at the $l \mathrm{~m}$ point from the cooling section inlet can be calculated by

$$
\dot{m}_{V}=\dot{m}_{V \_} h_{-} \text {outlet }-h \pi r^{2} l,
$$

where $\dot{m}_{V \_ \text {_outlet }}, h, r$, and $l$ are the mass flow rate of vapor at the heating section outlet, the heat transfer coefficient between the tube surface and the ambient air, the inner radius of a tube, and the distance from the cooling section inlet.

The void ratio, $e$, can be calculated by the total mass flow rate, $\dot{M}$, and the mass flow rate of vapor, $\dot{m}_{V}$, as follows, by taking into consideration that the difference between $\dot{M}$ and $\dot{m}_{V}$ is the mass flow rate of liquid:

$$
e=\frac{\dot{m}_{V} / \rho_{V}}{\left(\dot{M}-\dot{m}_{V}\right) / \rho_{L}+\dot{m}_{V} / \rho_{V}} .
$$

Here, $\rho_{V}$ and $\rho_{L}$ are the vapor density and the liquid density. While flowing in the cooling section, the void ratio, $e$, is gradually decreased until all vapor plugs are condensed.

The total mass flow rate, $\dot{M}$, can be calculated using Eq. (5) on the two simple assumptions: (i) suitable amount of liquid flows into the heating section so that the capillary tube is never dried out, and (ii) the void ratio being increased through the heating section becomes one just at the point of the heating section outlet.

$$
\dot{M}=\frac{13 Q_{\text {heat }}-\dot{M} C_{p L}}{L} .
$$

The total mass flow rate, $\dot{M}$, was calculated using the iterative method; $\dot{M}$ was gradually increased iteratively until $e$ at the heating section outlet becomes one. From Eqs. (2), (3), (4) and (5), the void ratio, $e$, at each unit volume along the turn (or the loop) at a steady state can be calculated.

The buoyancy $\overrightarrow{F_{\text {buoyancy }}}$ can be calculated by

$$
\overrightarrow{F_{\text {buoyancy }}}=-\rho_{l} \vec{g} V e,
$$

where $\rho_{l}, g$, and $V$ are the liquid density, the gravitational acceleration, and the unit volume. The body force $\overrightarrow{F_{\text {body }}}$ can be calculated using

$$
\overrightarrow{F_{\text {body }}}=\rho_{l} \vec{g} V(1-e) \text {. }
$$

The gravity effect on the working fluid in the adiabatic section can be neglected, because the adiabatic section is aligned horizontally (or normal to the gravity direction). The total force $\overrightarrow{F_{\text {total }}}$ on the working fluid flow is

$$
\overrightarrow{F_{\text {total }}}=\overrightarrow{F_{\text {buoyancy }}}+\overrightarrow{F_{\text {body }}} \text {. }
$$




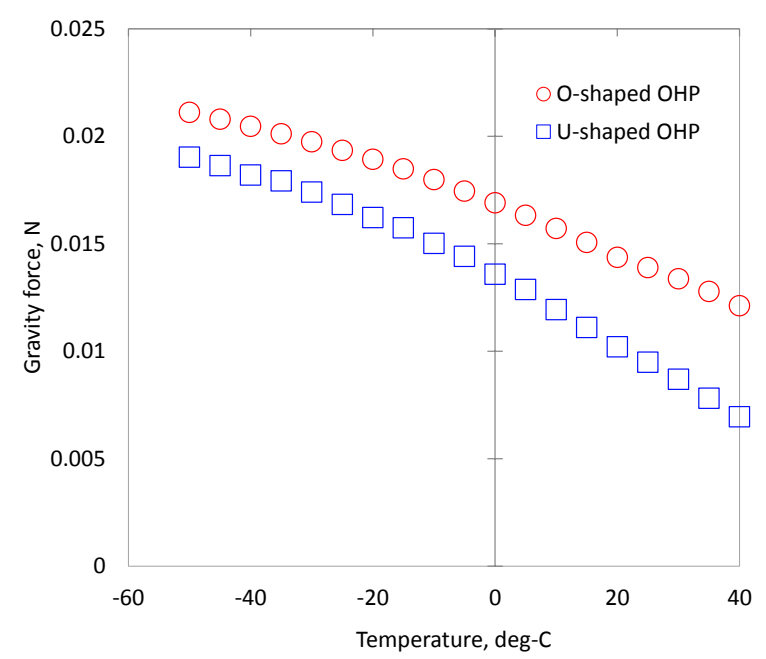

Fig. 15. The calculated gravity force assisting the working fluid flow per loop of the O-shaped OHP and per turn of the U-shaped OHP with $150 \mathrm{~W}$ heat load.

Check valve should be mounted onto the OHP with its forward direction in the positive direction of the vector $\overrightarrow{F_{\text {total }}}$. Figure 15 shows the calculated gravity $\underset{F}{\stackrel{f}{2}} F_{\text {int_total }}$, which is defined as the integrated $\overrightarrow{F_{\text {total }}}$ per U-shaped turn (or per O-shaped loop) in the case of $150 \mathrm{~W}$ heat load. At every temperature between $-60^{\circ} \mathrm{C}$ and $40^{\circ} \mathrm{C}$, the O-shaped OHP shows higher $F_{\text {int_total }}$ than the U-shaped OHP.

The above-mentioned calculations indicate that the O-shaped OHP can expect larger effect of the gravity assist than the U-shaped OHP, even though the U-shaped OHP needs larger drive force to obtain faster working fluid velocity $v$. This interpretation is in good qualitative agreement with the experimental results that the O-shaped OHP showed higher thermal performance than the U-shaped OHP.

\section{Summary}

Thermal performance of a large-scale U-shaped OHP model and its modified version of an O-shaped OHP model developed for GAPS has been investigated. It was experimentally verified that both the U-shaped and the O-shaped OHPs can operate in a wide temperature range between $20^{\circ} \mathrm{C}$ and $-60^{\circ} \mathrm{C}$ with sufficient thermal capability, which satisfies the GAPS design requirement at least in terms of the heat conductance. For instance, the thermal conductance of the U-shaped and the O-shaped OHP models were $10 \mathrm{~W} / \mathrm{K}$ and $12 \mathrm{~W} / \mathrm{K}$, respectively, at $-50^{\circ} \mathrm{C}$ with $150 \mathrm{~W}$ heat load. In comparison with the U-shaped model, generally the O-shaped model showed better thermal performance.

However, there is room for improvement especially at low temperatures. The maximum amount of the heat transport at low temperatures was around $150 \mathrm{~W}$, which has low margins from the design requirement. The heating section temperature was not uniform enough at low temperatures. The start-up time got longer at low temperatures and is desired to be shortened.

In order to seek a way to improve the OHP design, we tried to clarify the drive force to circulate the OHP working fluid, which causes the performance difference between the two OHP models. The calculations of the pressure loss in the capillary tube and the effect of the gravity assist showed good qualitative agreement with the experimental results that the O-shaped OHP can have higher thermal performance than the U-shaped OHP.

Based on these achievements, we will further develop the OHP design to make a more suitable heat transfer device for GAPS. The interpretation of the gravity effect is useful, for instance, to optimize the capillary tube diameter as well as to seek a more suitable working fluid material. Once this large-scale OHP is realized, the OHP is expected to be utilized for many applications with its great potential as a valuable thermal control device.

\section{Acknowledgments}

This work is partly supported by MEXT Grants KAKENHI (22340073 and 26707015).

\section{References}

Akachi, H., Polasek, H. \& Strulc, P. [1996] "Pulsating heat pipes," in Proceedings of the 5th International Heat pipe Symposium, (Royal Melbourne Inst. of Technology, Melbourne, Austria, 1996), pp. 208-217.

Fuke, H., Ong, R. A., Aramaki, T. et al. [2014] "The pGAPS experiment: An engineering balloon flight of prototype GAPS," Advances in Space Research 53, 1432-1437.

Hailey, C. J., Aramaki, T., Boggs, S. E. et al. [2013] "Antideuteron based dark matter search with GAPS: Current progress and future prospects," Advances in Space Research 51, 290-296.

Iwata, N., Ogawa, H. \& Miyazaki, Y. [2011] "Temperaturecontrollable oscillating heat pipe," Journal of Thermophysics and Heat Transfer 22(3), 386-392.

Mognet, S. A. I., Aramaki, T. \& Bando, N. et al. [2014] "The prototype GAPS (pGAPS) experiment," Nucl. Instr. and Methods A 735, 24-38. 
Nagashima, A. (ed). [2008] Thermophysical Properties Handbook, Japan Society of Thermophysical Properties Series (Yokendo, Tokyo Japan), pp. 446-448. U.S. Munitions List, sections 38 and $47(7)$ of the Arms Export Control Act 22 U.S.C. 2778 and 2794(7).

Okazaki, S., Fuke, H., Miyazaki, Y., Okubo, T. \& Ogawa, H. [2012] "Development of the cooling system for GAPS detectors," Space Science Symposium, P4-438, Jan. 2012 (in Japanese).

Okazaki, S., Fuke, H., Miyazaki, Y., Okubo, T. \& Ogawa, H. [2012] "Development of a meter-scale U-Shaped oscillating heat pipe for GAPS," in 42nd International Conference on Environmental Systems, 15-19 July 2012, San Diego, California, AIAA 2012-3499. 